\title{
MEASURING THE FRACTAL STRUCTURE OF INTERSTELLAR CLOUDS
}

M.G.R. VOGELAAR

Kapteyn Laboratory

Rijks Universiteit Groningen

Postbus 800

9700 AV Groningen

The Netherlands

\author{
B.P. WAKKER \\ Department of Astronomy \\ University of Illinois \\ $1002 \mathrm{~W}$ Green $S t$ \\ Urbana IL61801 \\ USA
}

\author{
U.J. SCHWARZ \\ Kapteyn Laboratory \\ Rijks Universiteit Groningen \\ Postbus 800 \\ 9700 AV Groningen \\ The Netherlands
}

ABSTRACT. To study the structure of interstellar clouds we used the so-called perimeter-area relation to estimate fractal dimensions. We studied the reliability of the method by applying it to artificial fractals and discuss some of the problems and pitfalls. Results for two different cloud types (high-velocity clouds (HVCs) and infrared cirrus) are summarized. We find dimensions $1.2<D<1.55$, somewhat higher than found in previous, similar studies.

\section{The Perimeter-Area Relation}

Mandelbrot's relation between area and perimeter of a closed fractal curve, $P(l)=$ $\rho A(l)^{D / 2}$, provides an algorithm to estimate the fractal dimension of interstellar clouds. The projection on the sky of the emission gives a brightness distribution and the brightness contours form the cloud perimeters. Within these clouds we define "objects" as sets of connected pixels with brightness above a given level. If clouds are real fractals we expect $1<D<2$. The factor $\rho$ is related to the shape of the cloud (Feder 1988) and is called the prefactor.

\section{Data}

Five HVC fields were measured (21-cm H I WSRT data, see e.g. Wakker \& Schwarz 1990). The original data are channel maps at $1^{\prime}$ resolution. For the measurements all channels were used together, as if they formed one big mosaiced map. We also used $100 \mu \mathrm{m}$ data from Laureijs (1989): the relatively isolated dark clouds L134, G240-66 and G102+70 and a field named "Ring" which may contain a supernova remnant. Further we used the $100 \mu \mathrm{m}$ maps of HVCs AIII /AIV and MI (Wakker \& Boulanger 1985) and an unpublished, $60^{\circ} \times 60^{\circ}$ map of the anticentre. The resulting dimensions and prefactors are summarized in Fig. 1.

\section{Problems and pitfalls}

There are some problems associated with using the perimeter-area relation.

-As all available data are on grids, perimeters can only be measured approximately. The value depends on the way corners are treated. Normally, this only influences the value of the prefactor. Furthermore, the gridding implies that a minimum number of pixels is needed to define an object. We found 25 pixels to be a good number.

- A severe problem is the presence of noise. At low levels and at levels close to the map maximum, the noise distorts the contours considerably. So, we excluded contours below 3 sigma and closer than 4 sigma to the peak. A preliminary test on the influence of the signal-to-noise on the derived dimension gave different results for an artificial fractal and a real observation. For an artificial fractal constructed using fractional Brownian motion ( $\mathrm{fBm})$ the estimate was much too high for signal-to-noise ratios below 50. Adding noise to a real observation gave a constant estimate for ratios above 15 .

-For the HVCs we assumed that the dimension is independent of the velocity interval. To test this, we measured $D$ for different intervals, containing a comparable number of 


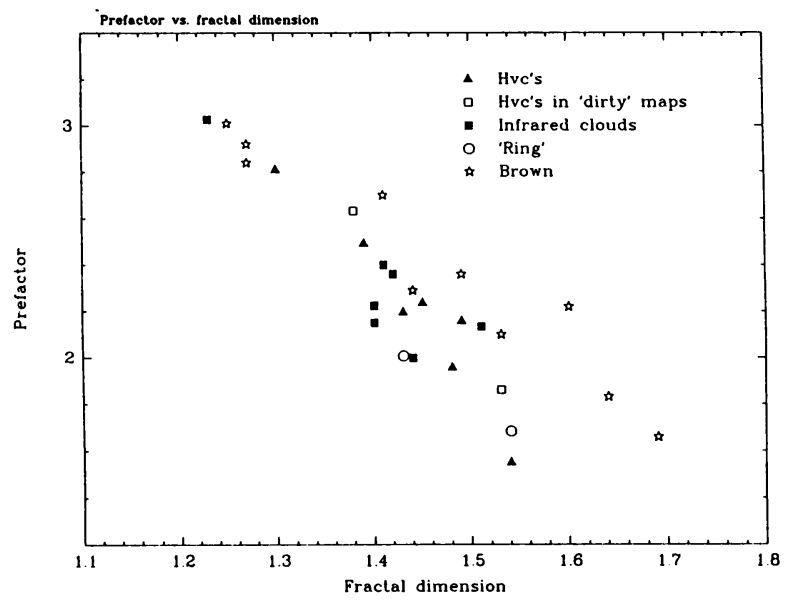

Figure 1. Estimated prefactor $\rho$ versus estimated fractal dimension $D$ for all fields.

objects. Probably because of low signal-to-noise in edge channels, the estimates then seem to depend on the chosen interval. This effect makes estimating dimensions for HVCs more difficult.

-A complication is posed by several projection effects. Firstly, we observe the projection of a three-dimensional cloud on a plane, not a slice through the cloud. It is not mathematically necessary that these two dimensions are related. From a physical point of view the interesting dimension is that of the slice. Some evidence from laboratory studies of turbulence suggests that the dimensions may indeed be different (Méneveau 1989). A second effect is that in one particular field there may be many overlapping clouds at different distances, each of which may have different intrinsic structure. A way to minimize this effect is to use velocity information to separate clouds. Finally, the cloud structure may be multi-fractal, i.e. consisting of intertwining structures with different fractal dimensions. A possible indication of the presence of multi-fractal structure can be found in the plots of area vs perimeter. A large scatter means there is not an easily definable single dimension.

\section{Prefactor}

All our maps had different gridspacings, so a correction must be applied to compare prefactors. The resulting values should be approached with care. Also, the value depends on the approximation used to construct the perimeter from horizontal, vertical or diagonal (at corners) segments (in the original derivation it was assumed that the ruler could have any orientation).

In principle $\rho$ can have any value for a given $D$. However, in our measurements, the corrected prefactor and the dimension appear related (Fig. 1). That the same relation is obtained for artificial clouds suggests it is an artifact of the method used.

\section{Conclusions}

The real reason for applying fractal geometry to the ISM is to understand the physical processes underlying its structure. Clouds subject to the same influences should have the same dimension. The connection with physics is not yet clearly understood, however. Some models of turbulence predict fractal dimensions in specific cases. Méneveau (1989) 
predicts $D=4 / 3$ from a model for turbulent flow. Hentschel \& Procaccia (1984) calculated from their theory of "relative turbulent diffusion" that $1.37<D<1.41$. The dimensions we found are usually higher than, but close to, these theoretical values; dimensions for infrared cirrus are a little closer to the predictions than those for HVCs, possibly because of the lower signal-to-noise ratio.

\section{References}

Falgarone E., 1989, Structure and Dynamics of the Interstellar Medium, eds. G. TenorioTagle, M. Moles, J. Melnick, IAU Colloquium 120, Springer Verlag, Berlin, p68

Feder J., 1988, Fractals, Plenum Press, New York/London

Hentschel H.G.E., Procaccia I. 1984, Phys. Rev. A., 29, 1461

Laureijs R., 1989, Ph. D. Thesis, University of Groningen

Méneveau, C., 1989, Ph. D. Thesis, Yale University

Wakker B.P., Boulanger F., 1986, Astron. Astrophys. 170, 84

Wakker B.P., Schwarz U.J., 1990, submitted to A\&A 